\title{
The Application of Grey-TOPSIS Method on Teaching Quality Evaluation of the Higher Education
}

\author{
http://dx.doi.org/10.3991/ijet.v10i8.5219 \\ Jing Song ${ }^{1}$, Junhui Zheng ${ }^{2}$ \\ ${ }^{1}$ College of Suqian, Jiangsu, China \\ ${ }^{2}$ PINGDINGSHAN University, Pingdingshan Henan, China
}

\begin{abstract}
The teaching quality of the higher school is not only related to the development of the students, but also related to the future of our country. It can find out the problems of the higher education for colleges to evaluate the teaching quality of the higher education. And it can provide the reference for the students to apply for the colleges. In this paper, we combine the grey correlation with TOPSIS method and provide the improved Grey-TOPSIS method. Then, we evaluate the teaching quality of the higher education. The results show that the comprehensive evaluation model can evaluate reasonably the teaching quality of the higher education. And it proves the validity and reliability of the method.
\end{abstract}

Index Terms-Evaluation, Grey correlation, Higher education.

\section{INTRODUCTION}

In recent years, with the continuous expansion of the college enrollment, the number of the college students is rising continuously. The rise of the number is bound to some difficulties for the management of the colleges. As the important part of the college management, the teaching quality of the higher education has been concerned by all aspects. Therefore, it is very necessary to evaluate the teaching quality of the higher education.

Pan Aizhen studied the evaluation behavior of the higher education that the Chinese government participated. The author thought that the evaluation model that Chinese government participated had some problems. And the author perfected it [1]. From the perspective of the new institutional economics, Liu Shuyun studied the reconstruction of the higher education evaluation system in China. And they did the related research [2]. Geng Guiying analyzed the disadvantages of the higher education evaluation. And he proved the countermeasures to improve the evaluation system of the higher education in China [3]. Wei Hong and Zhong Binlin thought that it was the new trend of the evaluation development to pay attention to the studying effect of the students. The trend provided the beneficial enlightenment for the development of the higher education in China and the quality assurance [4]. From the conflict between the quality and the quantity for the higher education in China, Wu Wenwei established the unique higher education evaluation system in China [5]. From the theory research of the education evaluation and the practice of the analysis level, Li Jingming discussed the situation for the higher education evaluation system in China and the development trend. In addition, he proposed some ideas that promoting and improving the higher education evaluation in China [6].

The Grey theory was proposed by Professor Deng Julong [7-8]. Grey correlation method was one of the important methods in the Grey theory [9-10]. The Grey correlation method was called the Grey correlation degree. As the method for measuring the correlation degree among the factors, it measured according to the similar degree or the dissimilar degree of the development tendency among the factors [11-12]. The Grey correlation method was applied in many aspects [13-15].

TOPSIS method ranks according to the distance between the evaluated object and the optimal solution or the evaluated object and the worst solution [16-18]. Then we can get the evaluated results. As one of the mainstream evaluation methods, TOPSIS method has been applied widely in many aspects. For example, it has applied in investment decision field [19-20] and performance evaluation field etc [21-22].

In this paper, we combine the grey correlation with TOPSIS method and provide the improved Grey-TOPSIS method. Then, we apply the method to evaluate the teaching quality of the higher education. The structure of this paper is as follows. The first part is the introduction. The second part is the Grey correlation model. In this part, we introduce the Grey correlation model. The third part is the improved Grey-TOPSIS method. In this part, we improve the improved Grey-TOPSIS method. The fourth part is the experiment and the last part is the conclusion.

\section{GREY CORRELATION MODEL}

We assume that system feature behavior sequence is,

$$
X_{0}(k)=\left(x_{0}(1), x_{0}(2), \mathrm{L}, x_{0}(n)\right)
$$

The related factors behavior sequence of the system is,

$$
X_{i}(k)=\left(x_{i}(1), x_{i}(2), \mathrm{L}, x_{i}(n)\right), \quad(i=1,2, \mathrm{~L}, \mathrm{~m})
$$

Then, we introduce some common correlation degree for calculating $X_{0}$ and $X_{i}$.

(1) Deng's correlation degree

Firstly, the correlation degree coefficient is,

$$
\xi_{0 i}(k)=\frac{\min _{i} \min _{k}\left|X_{0}(k)-X_{i}(k)\right|+\rho \max _{i} \max _{k}\left|X_{0}(k)-X_{i}(k)\right|}{\left|X_{0}(k)-X_{i}(k)\right|+\rho \max _{i} \max _{k}\left|X_{0}(k)-X_{i}(k)\right|}
$$


Then, the correlation degree is,

$$
\gamma\left(X_{0}, X_{i}\right)=\frac{1}{n} \sum_{i=1}^{n} \xi_{0 i}
$$

It is the first model to be proposed for calculating the Grey correlation degree in the Grey system theory. The calculation emphasizes the influence for the correlation degree of the distance among the points. $\rho$ is the distinguishing coefficient. In general, we take $\rho=0.5$.

The specific rule for taking values is as follows.

We take $\Delta_{v}$ as the mean value for all difference absolute values. That is,

$$
\Delta_{v}=\frac{1}{n \cdot m} \sum_{i=1}^{m} \sum_{k=1}^{n}\left|x_{0}(k)-x_{i}(k)\right|
$$

We take $\varepsilon_{\Delta}=\frac{\Delta_{v}}{\Delta_{\max }}$. Then, the value of $\rho$ is following.

When $\Delta_{\max }>3 \Delta_{v}, \varepsilon_{\Delta} \leq \rho \leq 1.5 \varepsilon_{\Delta}$.

When $\Delta_{\max } \leq 3 \Delta_{v}, 1.5 \varepsilon_{\Delta} \leq \rho \leq 2 \varepsilon_{\Delta}$.

The model focuses on the overall analysis. Firstly, $\rho$ can adjust the size of $\xi_{0 i}(k)$. And it can control its change interval. The lower bound value of $\xi_{0 i}(k)$ becomes bigger with the bigger of $\rho$. The bigger of the lower bound value shows that the interval becomes smaller. Then the resolution rate becomes lower and the resolution effect is not obvious. Secondly, we select different resolution coefficients according to the correlation analysis among the factors. When calculating, we take $\rho=0.5$ and can get the satisfactory resolution. Thirdly, when $\rho \rightarrow \infty$, we cannot do the correlation analysis. At the same time, all $\xi_{0 i}(k) \rightarrow 1$. That is, the correlation coefficient transformers into a point.

\section{THE IMPROVED GREY- TOPSIS METHOD}

We assume that there are $s$ experts participating in the decision. The expert set is $D=\left(d_{1}, d_{2}, \mathrm{~L} d_{s}\right)$. The weight of the expert $d_{k}$ is $\lambda_{k}$. Where, $0 \leq \lambda_{k} \leq 1, k=1,2, \mathrm{~L}, s$ and $\sum_{k=1}^{s} \lambda_{k}=1$. The evaluation set is $A=\left\{A_{1}, A_{2}, \mathrm{~L}, A_{m}\right\}$. And the weight is $\omega=\left(\omega_{1}, \omega_{2}, \mathrm{~L}, \omega_{n}\right)$.

We assume that there is the evaluation of the annual $A=\left\{A_{1}, A_{2}, \mathrm{~L}, A_{m}\right\}$ in the low carbon evaluation system. It contains $F=\left\{F_{1}, F_{2}, \mathrm{~L}, F_{m}\right\}$ evaluation indexes. Where, $M=\{1,2, \mathrm{~L}, m\}$ and $N=\{1,2, \mathrm{~L}, n\}$. We assume that the decision matrix is $X=\left(x_{i j}\right)_{m \times n} . x_{i j}$ is the attribute value of the $j$ attribute in the $i$ year. Where, $i \in M$ and $j \in N$. The evaluation steps which are based on the entropy weight-gray correlation-TOPSIS method are as follows.

The first step is the data processing. The data processing adopts the range statistics method. The processed matrix is $Y=\left(y_{i j}\right)_{m \times n}$. If the bigger numerical value is excellent, the processing method is as follows.

$$
y_{i j}=\frac{x_{i j}-\min _{i}\left\{x_{i j}\right\}}{\max \left\{x_{i j}\right\}-\min _{i}\left\{x_{i j}\right\}}
$$

If the smaller numerical value is excellent, the processing method is as follows.

$$
y_{i j}=\frac{\max \left\{x_{i j}\right\}-x_{i j}}{\max \left\{x_{i j}\right\}-\min _{i}\left\{x_{i j}\right\}}
$$

The second step is to determine the weights $\omega=\left(\omega_{1}, \omega_{2}, L, \omega_{n}\right)$ of the indexes according to the entropy weight method.

The third step is to calculate the normalized decision matrix $Z=\left(z_{i j}\right)_{m \times n}$. Where $z_{i j}=\omega \mathrm{g} y_{i j}, i \in M$ and $j \in N$.

The fourth step is to determine the positive ideal solution and the negative ideal solution of the weighted normalized decision matrix $Z=\left(z_{i j}\right)_{m \times n}$.

$$
Z^{+}=\left(z_{1}^{+}, z_{2}^{+}, \mathrm{L}, z_{n}^{+}\right)=\omega Z^{-}=\left(z_{1}^{-}, z_{2}^{-}, \mathrm{L}, z_{n}^{-}\right)=0
$$

Where, $Z_{j}^{+}=\max _{i} z_{i j}=\omega_{j}$ and $Z_{j}^{-}=\min _{i} z_{i j}=0$.

The fifth step is to calculate the Euclid distance $d_{i}^{+}$and $d_{i}^{-}$of the positive ideal solution $Z^{+}$and the negative ideal solution $Z^{-}$.

$$
\begin{aligned}
& d_{i}^{+}=\sqrt{\sum_{j=1}^{n}\left(z_{i j}-z_{j}^{+}\right)^{2}} \\
& d_{i}^{-}=\sqrt{\sum_{j=1}^{n}\left(z_{i j}-z_{j}^{-}\right)^{2}} \quad(i \in M)
\end{aligned}
$$

The sixth step is to calculate gray correlation coefficient matrix $R^{+}$and $R^{-}$from each scheme to the positive ideal solution $Z^{+}$and the negative ideal solution $Z^{-}$.

$$
\begin{gathered}
R^{+}=\left(r_{i j}^{+}\right)_{m \times n} \\
r_{i j}^{+}=\frac{\min _{i} \min _{j}\left|z_{j}^{+}-z_{i j}\right|+\rho \max _{i} \max _{j}\left|z_{j}^{+}-z_{i j}\right|}{\left|z_{j}^{+}-z_{i j}\right|+\rho \max _{i} \max _{j}\left|z_{j}^{+}-z_{i j}\right|}=\frac{\rho \omega_{j}}{\omega_{j}-z_{i j}+\rho \omega_{j}} \\
R^{-}=\left(r_{i j}^{-}\right)_{m \times n} \\
r_{i j}^{+}=\frac{\min _{i} \min _{j}\left|z_{j}^{-}-z_{i j}\right|+\rho \max _{i} \max _{j}\left|z_{j}^{-}-z_{i j}\right|}{\left|z_{j}^{-}-z_{i j}\right|+\rho \max _{i} \max _{j}\left|z_{j}^{-}-z_{i j}\right|}=\frac{\rho \omega_{j}}{z_{i j}+\rho \omega_{j}} \\
\rho \in(0, \infty) \text { is the distinguished coefficient. The }
\end{gathered}
$$
smaller the $\rho$ is, the bigger the distinguished capacity is. The interval value of $\rho$ is $(0,1)$. The specific value can be determined by the situation. When $\rho \leq 0.5463$, the distinguishability is the best. In general, $\rho=0.5$.

The seventh step is to calculate the gray correlation degree $r_{i}^{+}$and $r_{i}^{-}$from each scheme to the positive ideal solution $Z^{+}$and the negative ideal solution $Z^{-}$.

$$
r_{i}^{+}=\frac{1}{n} \sum_{j=1}^{n} r_{i j}^{+}, r_{i}^{-}=\frac{1}{n} \sum_{j=1}^{n} r_{i j}^{-}
$$


The eighth step is to make the dimensionless processing for $d_{i}^{+}, d_{i}^{-}, r_{i}^{+}$and $r_{i}^{-}$in the step five and the step seven. Then we get $D_{i}^{+}, D_{i}^{-}, R_{i}^{+}$and $R_{i}^{-}$.

$$
\begin{gathered}
D_{i}^{+}=\frac{d_{i}^{+}}{\max _{i} d_{i}^{+}} D_{i}^{-}=\frac{d_{i}^{-}}{\max _{i} d_{i}^{-}} \\
R_{i}^{+}=\frac{r_{i}^{+}}{\max _{i} r_{i}^{+}} R_{i}^{-}=\frac{r_{i}^{-}}{\max _{i} r_{i}^{-}}(i \in M)
\end{gathered}
$$

The ninth step is to merge the dimensionless distance and the correlation in the step eight. The bigger the $D_{i}^{-}$ and $R_{i}^{+}$are, the more approximate to the positive ideal solution the scheme is. The bigger the $D_{i}^{+}$and $R_{i}^{-}$are, the much far away from the positive ideal solution the scheme is. We assume that $S_{i}^{+}=\alpha D_{i}^{-}+\beta R_{i}^{-}$and $S_{i}^{-}=\alpha D_{i}^{-}+\beta R_{i}^{+}(i \in M)$. Where, $\alpha$ and $\beta$ reflect the preference of the location and the shape. And they meet $\alpha+\beta=1$ and $\alpha, \beta \in(0,1)$. The decision makers can determine their values according to their own preferences. $S_{i}^{+}$reflects synthetically the approaching degree between the scheme and the ideal scheme. The bigger the value is, the optimal the scheme is. $S_{i}^{-}$reflects synthetically the far away degree between the scheme and the ideal scheme. The bigger the value is, the bad the scheme is.

The tenth step is to the relative closeness degree $C_{i}^{+}=S_{i}^{+} /\left(S_{i}^{+}+S_{i}^{-}\right)$of the scheme. where, $(i \in M)$. We rank them according to the value of the relative closeness degree. The bigger the relative closeness degree is, the optimal the scheme is. The smaller the relative closeness degree is, the bad the scheme is.

The eleventh step is as follows. After we get the evaluation scheme, we adjust the weights. We assume that,

$$
\begin{aligned}
& x_{k}(i)=\sum_{j=1}^{m} x_{i j}^{k} \omega_{n} \\
& x_{0}(i)=\sum_{k=1}^{s} x_{k} \lambda_{k}
\end{aligned}
$$

Then, we calculate the correlation coefficient among $x_{k}$. We take it as follows.

$$
\xi_{0 k}(l)=\frac{m+M \eta}{\Delta_{0 k}(l)+M \eta}
$$

Where,

$$
\Delta_{0 k}(l)=\left|x_{0}(l)-x_{k}(l)\right|, m=\min _{k} \min _{l} \Delta_{0 k}(l), M=\max _{k} \max _{l} \Delta_{0 k}(l) .
$$

And, $\eta \in[0,1]$ is the distinguished coefficient and is a fixed constant in advance.

The twelfth step is to calculate the gray correlation degree among $x_{k}$. And we take it as follows.

$$
R_{0 k}=\frac{1}{n} \sum_{l=1}^{n} \xi_{0 k}(l)
$$

The thirteenth step is to adjust the weight of the expert. In order to prevent to purse the consistency of the opinion and ignore the effect of the results for the experts, we need to adjust the weight of the experts for the expert themselves.

$$
\lambda_{k}^{\prime}=\lambda_{k} R_{0 k} \sum_{k=1}^{s} / \lambda_{k} R_{0 k}
$$

According to adjust the weights of the experts, we calculate the new parameters $x_{0}^{\prime}(i)$. And we define the distance,

$$
L\left(x_{0}, x_{0}^{\prime}\right)=\sqrt{\sum_{i=1}^{n}\left(x_{0}(l)-x_{0}^{\prime}(l)\right)^{2}}
$$

We assume that the threshold is $r$. If $L\left(x_{0}, x_{0}^{\prime}\right) \leq r$ and the error of two results is smaller, we think that the decision results are stable and the adjustment process is over. And we take $x_{0}^{\prime}$ as the final evaluation results. Otherwise, we make $\lambda_{k}=\lambda_{k}^{\prime}, x_{0}=x_{0}^{\prime}$ and use the above method adjust the weights of the experts.

The fourteenth step is to get the new ranking result according to the adjusted weights of the experts.

$$
C=C_{i 1}^{+} \lambda_{1}+C_{i 2}^{+} \lambda_{2}+\mathrm{L}+C_{i k}^{+} \lambda_{k}
$$

\section{EXPERIMENT}

In this paper, we apply the improved Grey-TOPSIS method to evaluate the teaching quality of the higher education. In order to evaluate the teaching quality of the higher education, firstly, we need to establish the evaluation system. The teaching quality evaluation system of the higher education is as follows.

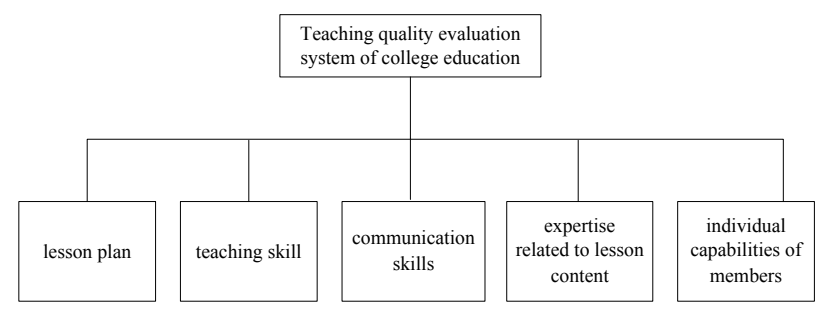

Figure 1. Teaching quality evaluation system of the higher education

We invite five experts to evaluate the teaching quality of the higher education. Firstly, we determine the weights of the attributes and the weights of the experts. They are $\omega=(0.2823,0.1962,0.1934,0.1657,0.1624)$ and

$\lambda=(0.2211,0.2034,0.1876,0.1933,1946)$. Then, we invite the first expert to evaluate. According to the data processing, we can get,

$$
\begin{aligned}
& r^{+}=\{0.4811,0.5814,0.6617,0.6341,0.8025\} \\
& r^{-}=\{0.8843,0.6256,0.5107,0.4500,0.4788\}
\end{aligned}
$$

Then, we do the non-dimensional processing,

$$
\begin{aligned}
D^{+} & =\{0.9986,0.7344,0.6362,0.5794,0.5523\} \\
D^{-} & =\{0.5478,0.6239,0.7854,0.8635,0.9887\} \\
R^{+} & =\{0.5998,0.6643,0.7565,0.8271,1.0002\} \\
R^{-} & =\{1.0003,0.7856,0.6625,0.5981,0.6102\}
\end{aligned}
$$

We assume $\alpha=\beta=0.5$ and we get the relative closeness degree.

$$
C_{i 1}^{+}=\{0.3766,0.4672,0.5986,0.5311,0.6404\}
$$

Then, we adjust the weights of the experts. And we get the new weights of the experts.

$$
\lambda=(0.2204,0.2012,0.1894,0.1987,1903)
$$


We set the threshold $r=0.003$. If $L\left(x_{0}, x_{0}^{\prime}\right)>0.003$, we need to adjust the weight. After the adjustment of three times, we get the new weights of the experts.

$$
\lambda=(0.2158,0.1937,0.2179,0.1904,1822)
$$

According to $C=C_{i 1}^{+} \lambda_{1}+C_{i 2}^{+} \lambda_{2}+\mathrm{L}+C_{i k}^{+} \lambda_{k}$, we get the new relative closeness degree,

$$
C=\{0.4688,0.3590,0.6325,0.5863,0.6204\}
$$

Therefore, the evaluation results of the teaching quality of the higher education for the five colleges are,

$$
\begin{gathered}
f_{3}>f_{5}>f_{4}>f_{1}>f_{2} \\
\text { V. CONCLUSIONS }
\end{gathered}
$$

\section{CONCLUSIONS}

Colleges are the place to train the talents. According to the higher education, the colleges transport many talents for the society. Evaluating the teaching quality of the higher education can improve the teaching level of the higher school. This paper applies the improved GreyTOPSIS method to evaluate the teaching quality of the higher education. This paper has the following works. Firstly, this paper introduces the Grey correlation model. Secondly, this paper proposes the improved Grey-TOPSIS method. Thirdly, this paper applies the improved GreyTOPSIS method to evaluate the teaching quality of the higher education. The experimental results demonstrate the validity and the reliability of the method.

\section{REFERENCES}

[1] Pan Aizhen. On the action of the Chinese government's participation in the assessment of higher education [D]. East China Normal University, Higher education, 2008.

[2] Liu Shuyun. Study on reconstruction of the system of higher education evaluation -based on the new institutional economics [D]. East China Normal University, Senior Executive Education, 2014.

[3] Geng Guiying. Research on higher education evaluation of China [D]. Nanjing University of Aeronautics and Astronautics, Theory and practice of administrative management, 2012.

[4] Wei Hong, Zhong Binlin. The new trend of international higher education evaluation and Its Enlightenment [J]. Higher education in China, 2009, 10: 16-19.

[5] Wen Wenwei. Study on higher education quality evaluation in China [D]. Jilin University, Ideological and political research, 2013.

[6] Li Jingming. Research on the improvement of high education evaluation system [D]. Soochow University, Higher education, 2008.

[7] Migdat Hodzic, Li-Chou Tai. Grey Predictor reference model for assisting particle swarm optimization for wind turbine control [J]. Renewable Energy, 2016, 86 251-256. http://dx.doi.org/10.1016/ j.renene.2015.08.001

[8] Hongjun Li, Ching Y. Suen. A novel Non-local means image denoising method based on grey theory $[\mathrm{J}]$. Pattern Recognition, 2016, 49: 237-248. http://dx.doi.org/10.1016/j.patcog.2015.05.028

[9] Xiaoliang Jia, Haizhong An, Wei Fang, Xiaoqi Sun, Xuan Huang. How do correlations of crude oil prices co-move? A grey correlation-based wavelet perspective $[\mathrm{J}]$. Energy Economics, 2015, 49: 588-598. http://dx.doi.org/10.1016/j.eneco.2015.03.008

[10] Ali Charbal, John-Eric Dufour, Adrien Guery, François Hild, Stéphane Roux, Ludovic Vincent, Martin Poncelet. Integrated Digital Image Correlation considering gray level and blur variations: Application to distortion measurements of IR camera [J]. Optics and Lasers in Engineering, 2016, 78: 75-85. http://dx.doi.org/10.1016/j.optlaseng.2015.09.011

[11] Liu Sifeng, Cai Hua, Yang Yingjie, Caoying. Advance in grey incident analysis modeling [J]. System Engineering- Theory \& Practice, 2013, 8: 2041-2046.
[12] Yuan Yuan, Qin Zhan, Jianyong Huang, Jing Fang, Chunyang Xiong. Digital image correlation with gray gradient constraints: Application to spatially variant speckle images [J]. Optics and Lasers in Engineering, 2016, 77: 85-91. http://dx.doi.org/10.1016/ j.optlaseng.2015.07.012

[13] Feng Xie, Zhen Chen, Jiaxing Shang, Geoffrey C. Fox. Grey Forecast model for accurate recommendation in presence of data sparsity and correlation [J]. Knowledge-Based Systems, 2014, 69: 179-190. http://dx.doi.org/10.1016/j.knosys.2014.04.011

[14] Zhenjun Wang, Qiong Wang, Tao Ai. Comparative study on effects of binders and curing ages on properties of cement emulsified asphalt mixture using gray correlation entropy analysis [J]. Construction and Building Materials 2014, 54: 615-622. http://dx.doi.org/10.1016/j.conbuildmat.2013.12.093

[15] Dongfeng MAO, Menglan DUAN, Xinzhong LI, Junwei SU, Yingying WANG. Selection of deepwater floating oil platform based on grey correlation $[\mathrm{J}]$. Petroleum Exploration and Development, 2013, 40(6): 796-800. http://dx.doi.org/10.1016/ S1876-3804(13)60107-X

[16] Yokabed Beikkhakhian, Mohammad Javanmardi, Mahdi Karbasian, Bijan Khayambashi. The application of ISM model in evaluating agile suppliers selection criteria and ranking suppliers using fuzzy TOPSIS-AHP methods [J]. Expert Systems with Applications, 2015, 42(15-16): 6224-6236. http://dx.doi.org/10.10 16/j.eswa.2015.02.035

[17] Ludmila Dymova, Pavel Sevastjanov, Anna Tikhonenko. An interval type-2 fuzzy extension of the TOPSIS method using alpha cuts $[\mathrm{J}]$. Knowledge-Based Systems, 2015, 83: 116-127. http://dx.doi.org/10.1016/j.knosys.2015.03.014

[18] Halim Kazan, Engin Karaman, Burçay Yaşar Akçalı, Elçin Şişmanoğlu. Assessment of TEOG Examination Success: Topsis Multi-Criteria Decision-Making Method Practice [J]. Procedia Social and Behavioral Sciences, 2015, 195: 915-924. http://dx.doi.org/10.1016/j.sbspro.2015.06.371

[19] [19] Zhang Yina. Study on the combination of the TOPSIS optimization and Grey relevancy in the real estate investment decision-making [D]. Xi'an University Of Architecture And Technology. Engineering Economy \& Management, 2008.

[20] Guo Yanfei. Research on venture capital projects evaluation and selection of high- tech enterprises in China [D]. Beijing University of Chemical Technology, Technology economy and management, 2008.

[21] Lu Chunyang, Wen Feng, Yang Qiongyuan, Chen Linlin, Zong Huiming. An evaluation of urban land use performance based on the improved TOPSIS method and diagnosis of its obstacle indicators: A case study of Chongqing [J]. Research Science, 2011, 33: 535-541.

[22] Li Can, Zhang Fengrong, Zhu Taifeng, Feng Ting, An Pingli. Evaluation and correlation analysis of land use performance based on entropy-weight TOPSIS method [J]. Transactions of the Chinese Society of Agricultural Engineering, 2013, 3: 217-227.

\section{AUTHORS}

Jing Song is an instructor and lecturer at School of Law and Politics of Suqian College, Jiangsu, 223800, China. Her research fields are higher education and ideological and political education. (email: songjina@163.com)

Junhui Zheng received his M.Sc. in Information Sciences (2010) from University. Now he is lecturer of informatics at College of computer science and technology, PINGDINGSHAN University, Pingdingshan Henan, China, 467000. His current research interests include Artificial Intelligence and Control Techniques. (email: pdszhengjunhui@163.com)

This paper was supported by 2014 Key Research Fund of SchoolLevel Higher Education Research of Suqian College: Study on Employment Difficulties and Count measures of Students in Higher Learning Institutions. (Grant No. 2014GJ02). Submitted 07 November 2015. Published as resubmitted by the authors 05 December 2015. 